\title{
Chlorthalidone for Poorly Controlled Hypertension in Chronic Kidney Disease: An Interventional Pilot Study
}

\author{
Rajiv Agarwal Arjun D. Sinha Maria K. Pappas Farah Ammous \\ Indiana University School of Medicine and Richard L. Roudebush Veterans Administration Medical Center, \\ Indianapolis, Ind., USA
}

\section{Key Words}

Chronic kidney disease · Blood pressure · Estimated GFR ·

Chlorthalidone

\begin{abstract}
To test the hypothesis that thiazide-type diuretics effectively lower blood pressure (BP) in moderate to advanced chronic kidney disease (CKD; estimated GFR 20-45 $\mathrm{ml} / \mathrm{min} /$ $1.73 \mathrm{~m}^{2}$ ), after confirming poorly controlled hypertension with 24-hour ambulatory BP monitoring, chlorthalidone was added to existing medications in a dose of $25 \mathrm{mg} /$ day, and the dose doubled every 4 weeks if the BP remained elevated. The average age of the 14 subjects was 67.5 years, a median of 4 antihypertensive drugs were used and estimated GFR was $26.8 \pm 8.8 \mathrm{ml} / \mathrm{min} / 1.73 \mathrm{~m}^{2}$. Twelve subjects completed the 12-week treatment phase, and the 24-hour BP, which was $143.1 / 75.1 \mathrm{~mm} \mathrm{Hg}$ at baseline, was reduced by $10.5 /$ $3.1 \mathrm{~mm} \mathrm{Hg}(p=0.01 / p=0.17)$. Home BP prior to initiating chlorthalidone was $152.4 / 82.6 \mathrm{~mm} \mathrm{Hg}$ and fell at 4,8 , and 12 weeks by 10.2/4.8, 13.4/6.0, and 9.4/3.7 $\mathrm{mm} \mathrm{Hg}$ (all $p<0.05$ ). Maximal reduction in body weight and total body volume (measured by air displacement plethysmography) was seen at 8 weeks, concurrent with the maximal elevation in serum creatinine concentration and plasma renin activity. Albu-
\end{abstract}

minuria was significantly reduced by $40-45 \%$. Adverse events were seen following chlorthalidone therapy in 7 subjects who experienced 18 events as follows: hypokalemia $(n=4)$, hyperuricemia (4), hyponatremia (3), transient creatinine changes (3), dizziness (2), hyperglycemia (1), and constipation (1). One subject had ischemic stroke during the study. In conclusion, among people with moderate to advanced CKD with poorly controlled hypertension, chlorthalidone may significantly reduce BP via volume contraction; a randomized trial is needed to define the risks and benefits. Adverse effects may occur within a few weeks and should be carefully monitored.

(c) 2014 S. Karger AG, Basel

\section{Introduction}

It is estimated that in the United States there are approximately 8 million individuals with moderate to severe chronic kidney disease (CKD) not on dialysis [1]. Among these patients, volume expansion plays an important role in the pathogenesis of hypertension [2].

According to the most recent guidelines, diuretics should be used to treat hypertension in most patients with CKD [3]. For those with estimated GFR $\geq 30 \mathrm{ml} / \mathrm{min} /$

\section{KARGER}

E-Mail karger@karger.com

www.karger.com/ajn
(C) 2014 S. Karger AG, Basel

0250-8095/14/0392-0171\$39.50/0
Rajiv Agarwal, MD, Professor of Medicine

Indiana University and VAMC

1481 West 10th Street

Indianapolis, IN 46202 (USA)

E-Mail ragarwal@iu.edu 
$1.73 \mathrm{~m}^{2}$, thiazide diuretics given once daily are recommended. However, loop diuretics are recommended for those with estimated GFR $<30 \mathrm{ml} / \mathrm{min} / 1.73 \mathrm{~m}^{2}$. Loop diuretics given once or twice daily, in combination with thiazide diuretics, can be used for patients with extracellular fluid volume expansion and edema. All these recommendations have an A level of evidence. Joint National Commission 7 guidelines [4] state, 'with advanced renal disease (estimated GFR $<30 \mathrm{ml} / \mathrm{min} 1.73 \mathrm{~m}^{2}$, corresponding to a serum creatinine of $2.5-3 \mathrm{mg} / \mathrm{dl}$ ), increasing doses of loop diuretics are usually needed in combination with other drug classes'. Furthermore, the guidelines state, 'most patients with CKD should receive an ACEI or an $A R B$ in combination with a diuretic', and that many will require a loop diuretic rather than a thiazide.

Prior to conducting this pilot study, we conducted a systematic review and found 7 studies evaluating the use of thiazide diuretics in advanced CKD either alone or in combination with loop diuretics [5]. Of these, 3 studies were observational, 2 were single-blind, crossover studies, and 2 were double-blind crossover trials. We concluded that whether thiazides can lower blood pressure (BP) in patients with more advanced CKD remains unclear. Given the central role of volume excess in the pathogenesis of hypertension in CKD, and the low cost of thiazide diuretics, there is a need to study the use of these drugs to lower BP among patients with uncontrolled hypertension and moderately advanced CKD.

Accordingly, we hypothesized that among patients with moderate to severe $\mathrm{CKD}$, chlorthalidone will result in improved 24-hour ambulatory BP over 12 weeks. The sample size was chosen to provide preliminary data to inform design of a randomized controlled trial to improve control rates of hypertension in the CKD population.

\section{Methods}

\section{Study Design}

A single-center pilot study of chlorthalidone in patients with poorly controlled hypertension and CKD was designed as outlined in the supplementary figure 1 (for all supplementary material, see www.karger.com/doi/10.1159/000358603). The study protocol was approved by the Institutional Review Board and the VA Research and Development Committee, and all subjects provided written informed consent. The study was registered at clinicaltrials.gov (NCT01750294).

\section{Inclusion and Exclusion Criteria}

Eligible subjects were at least 18 years of age and at the time of recruitment had an estimated GFR $\leq 45 \mathrm{ml} / \mathrm{min} / 1.73 \mathrm{~m}^{2}$ but $>20$ $\mathrm{ml} / \mathrm{min} / 1.73 \mathrm{~m}^{2}$ using an IDMS-calibrated creatinine assay [6].
Poorly controlled BP was defined as $\geq 135$ systolic or $\geq 85 \mathrm{~mm} \mathrm{Hg}$ by 24 -hour ambulatory BP monitoring. Initially, the study was designed to study those with resistant hypertension, but due to difficulties with recruitment, we amended the protocol to study those with poorly controlled but treated hypertension. Prescription of a loop diuretic at baseline was preferred but not required. However, we required that one of the nondiuretic drugs be either an ACE inhibitor or angiotensin receptor blocker. If these were contraindicated, then use of a beta-blocker was required. We excluded subjects who used thiazide or thiazide-like drugs in the previous 3 months, were prescribed furosemide in a dose $>80 \mathrm{mg} /$ day, or had ambulatory BP that was deemed unsafe to participate ( $\geq 160$ systolic or $\geq 100 \mathrm{~mm} \mathrm{Hg}$ diastolic by 24-hour ambulatory BP monitoring). Other exclusions are listed in the online supplementary appendix.

\section{Study Intervention}

Subjects with CKD and poorly controlled hypertension confirmed by ambulatory BP monitoring after the 2-week run-in who met the inclusion and exclusion criteria were administered chlorthalidone in increasing doses. There are 8 study-related visits at weeks $-3,-2,0,4,8$, and 12 . At weeks 0 and 12 , visits were needed on consecutive days to evaluate 24-hour ambulatory BP and urine collection.

Subjects were identified in the renal clinic on the basis of estimated GFR. If they were on BP medications including a diuretic and thought to have poorly controlled hypertension, they were dispensed a home BP monitor. If home BP monitoring was $>130 / 80$ $\mathrm{mm} \mathrm{Hg}$, they were studied further.

At the beginning of the run-in, all subjects received a standard regimen of lisinopril $20-40 \mathrm{mg} /$ day, amlodipine $10 \mathrm{mg} /$ day, and torsemide $10-20 \mathrm{mg} /$ day. These doses were selected based on the existing regimen of the subject. For those on clonidine or minoxidil as fourth and fifth drugs, the doses of these drugs were left unchanged. Some subjects received alpha-blockers for prostatic symptoms. The dose of alpha-blockers was also not changed.

Chlorthalidone was selected over hydrochlorothiazide since it has a longer half-life (40-60 vs. $2.5 \mathrm{~h}$ in those with normal renal function) and potentially greater BP-lowering effects. It is generic and can be administered once daily.

Instead of a fixed dose study, we designed a forced-titration study because of uncertainty in the dose at which chlorthalidone may lower BP. Since BP-lowering effects may vary according to the GFR, the dose at which chlorthalidone improves BP was individualized according to response. Other antihypertensive agents were held constant during the intervention phase.

Goal BP was defined by home BP monitoring. At least 6 readings were needed to make a decision with home BP. We asked subjects to provide $14 \mathrm{BP}$ readings in 1 week. The average of these was used for decision to titrate the drug. However, if $<6$ home BP readings were available, we made decisions based on clinic BP.

Chlorthalidone dose was not increased if the subject had any of the following: home systolic BP of $<125 \mathrm{~mm} \mathrm{Hg}$, symptomatic hypotension, or other drug-attributable adverse event. In these instances, the dose of the drug was maintained or downtitrated.

\section{Procedures}

Ambulatory BP monitoring over $24 \mathrm{~h}$ was performed in all subjects at baseline and at the end of the 12 weeks of treatment using 
the Spacelabs 90207 monitor (see online suppl. appendix for details) [7].

Home BP measurements were performed with a home digital sphygmomanometer with an automatic inflator and printer for recording (Model HEM790IT, Omron Healthcare Inc., Bannockburn, Ill., USA) with a cuff size appropriate for arm size. Subjects were asked to record their BP after 5 min of seated rest in triplicate twice daily for 1 week prior to clinic visit. All subjects were instructed in use of the monitor prior to participation in the study.

Clinic BP measurements were obtained in triplicate in both arms, with the arm and the forearm supported at the level of the heart (mid-sternal level), by a trained observer after a 5-min rest using a digital sphygmomanometer (Model HEM-907, Omron Healthcare) with an appropriate cuff size using oscillometric methodology as stated in the European Society of Hypertension recommendations for BP measurement [8]. Oscillometric BP averaged over three readings was used as that clinic visit BP. After the first visit, the arm with the higher systolic BP was designated the BP arm for the duration of the study.

Concentration of urinary albumin and creatinine concentration was determined using clinical assays in the hospital laboratory. Measurements were made in 24-hour urine collected during sleep and wake states in separate aliquots.

Body volume was measured using air displacement plethysmography (ADP) using the BOD POD Gold Standard Body Composition Tracking System (Life Measurement Inc., Concord, Calif., USA). The ADP system consisted of the air plethysmograph, a digital scale, and computer software (BOD POD version 4.2+). Each participant was asked to change into compression shorts and a swim cap and to remove any jewelry. Body mass was measured to the nearest $0.001 \mathrm{~kg}$ using the electronic scale prior to the body volume measurement. Height was measured using a Seca $222 \mathrm{mea}-$ suring rod (Seca Group, Hamburg, Germany).

Pulse wave shape and velocity were measured via the SphygmoCor EM3. Central aortic pressure and larger artery stiffness were recorded.

\section{Outcome and Safety}

The primary outcome variable in this trial was the change from baseline to 12 weeks in systolic ambulatory BP in the chlorthalidone group. Safety of continued participation was assessed at each study visit. Prespecified stop points were treatment-emergent severe adverse event that precluded further exposure to the drug such as an allergic drug reaction, initiation of renal replacement therapy or home BP $>160 / 100 \mathrm{~mm} \mathrm{Hg}$ confirmed at repeat visit within 2 weeks.

\section{Statistical Methods}

Data were plotted to examine individual level information. After thorough evaluation of data integrity, 24-hour ambulatory BP data were analyzed. Hourly averages were first calculated. Subsequently, each hourly BP was averaged to obtain the 24-hour BP. The primary method of data analysis was by mixed models [9]. Outcome variable was the 24-hour ambulatory systolic BP. Subject was used as a random variable, and visit was the fixed categorical variable. The statistical significance of change from baseline after fitting the model was calculated using Wald tests. Similar models were used to assess volume and weight. Plasma renin activity, aldosterone, B-type natriuretic peptide, and albumin/creatinine ra- tio were log-transformed to approximate a normal distribution. Statistical analysis was performed on these transformed variables. With respect to the transformed variables, the modeled means and their $95 \%$ confidence intervals were reported in the original units. The change from baseline of these transformed variables is therefore interpreted as fold change from baseline.

\section{Results}

Subjects were recruited from the renal clinic at Richard L. Roudebush VA Medical Center, Indianapolis, Ind., USA, between September 18, 2012, and May 15, 2013. The last subject completed the last visit on August 8, 2013.

Six hundred and two patients were screened, out of which 548 did not meet the initial eligibility criteria. Of the 52 eligible subjects, 39 consented and 34 underwent baseline home BP monitoring (at week -2); 5 did not show up for the initial visit. One subject was excluded because of too low a BP at home and 3 others were not adherent with baseline procedures and therefore could not be further studied. Of the 30 remaining subjects, BP medication changes were made, and subsequently ambulatory BP was found to be normal in 13 and too high in 3; thus, 14 remained eligible for further participation.

Of the 14 subjects who took at least one dose of the study drug, 2 did not complete the study. One withdrew consent because he no longer wanted to take the study medication after 2 days of treatment. One subject had to be excluded per protocol 2 weeks after treatment when home BP was noted to be persistently elevated ( $>160 / 100$ $\mathrm{mm} \mathrm{Hg}$ ) despite chlorthalidone. The remaining 12 completed 12 weeks of chlorthalidone therapy.

Baseline characteristics of the 14 subjects who took at least one dose of chlorthalidone was as follows: age 67.5 \pm 10.2 years (range 43-86); men (14); race: white (6), black (8); diabetes mellitus (12); insulin use (7); oral hypoglycemic drug use (6); current tobacco use (2); current alcohol use (4), and sleep apnea (6) which was treated with continuous positive airway pressure device in 3 . Vascular disease was extensive: past myocardial infarction (4); coronary revascularization (5); stroke (3); peripheral revascularization (1), and hospitalization for heart failure (2). All subjects had treated but poorly controlled hypertension and at the end of the run-in period were on a mean of $3.8 \pm 1.3$ drugs (median 4 , range 1-6), which included the following medications: ACE inhibitor or angiotensin receptor blocker (11); beta-blocker (11); calcium channel blocker (12); loop diuretic (9); alphablocker (2); spironolactone (3); central agents (3), and vasodilator (1). Baseline estimated GFR after run-in was 
$26.8 \pm 8.8 \mathrm{ml} / \mathrm{min} / 1.73 \mathrm{~m}^{2}$, and stage $3 \mathrm{~B}$ CKD was present in 4 , stage 4 in 8 , and stage 5 in 2 subjects. The latter 2 had stage $4 \mathrm{CKD}$ before medications were adjusted. The 2 subjects who dropped out had stage $4 \mathrm{CKD}$. Urine was collected during wake and sleep hours. Median baseline urine albumin excretion rate during waking hours was $923 \mathrm{mg} / \mathrm{g}$ creatinine (IQR 563-2,556) and during sleep it was $873 \mathrm{mg} / \mathrm{g}$ creatinine (IQR 477-3,037).

\section{Chlorthalidone Dosing}

At baseline, all subjects $(n=14)$ were prescribed 25 $\mathrm{mg} /$ day of chlorthalidone. At 4 weeks, 2 subjects were no longer taking the drug; in 1 , the dose was lowered to thrice weekly, in 6 it was not escalated, and in 5 it was titrated to $50 \mathrm{mg} /$ day. At 8 weeks, 1 subject had quit all medications, 1 was continued on chlorthalidone $25 \mathrm{mg}$ thrice weekly, 2 subjects on $25 \mathrm{mg} /$ day, 5 subjects on $50 \mathrm{mg} /$ day, and 3 were escalated to $100 \mathrm{mg} /$ day. At the final visit, 1 subject was continued on $25 \mathrm{mg}$ thrice weekly, 2 on $25 \mathrm{mg} /$ day, 6 on $50 \mathrm{mg} / \mathrm{day}$, and $2 \mathrm{on} 100 \mathrm{mg} /$ day. Mixed modeled mean prescribed chlorthalidone dosage at 4 weeks was 34 $\mathrm{mg}$, at 8 weeks $55 \mathrm{mg}$, and at final visit $51 \mathrm{mg} /$ day.

\section{Ambulatory BP Response}

Figure 1 shows the individual level and the mean 24hour, awake, and sleep systolic and diastolic BP in all participants. One subject stopped taking most antihypertensive medications including the study drug and had an increase in BP. The change from baseline in mean 24-hour systolic BP was significant $(10.5 \mathrm{~mm} \mathrm{Hg}$ decline, $\mathrm{p}=0.01$ ) whether the analysis was performed for 14 subjects or limited to 12 subjects who had paired measurements (decline $8.5 \mathrm{~mm} \mathrm{Hg}, \mathrm{p}=0.05$ ). Similar declines were seen in awake and sleep systolic BP, but the dipping ratio was not altered. Diastolic BP did not decline significantly.

\section{Home BP Response}

The time course of home BP is shown in figure 2. Week -2 represents the baseline BP after which the usual medi- cations were adjusted to a standard antihypertensive regimen. Two weeks after adjustment, at week 0 , chlorthalidone was initiated in a dose of $25 \mathrm{mg}$ once daily. All changes in either systolic or diastolic BP were statistically significant when measured either as change from baseline or change after drug initiation.

\section{Clinic BP and Orthostatic Response}

The time course of clinic BP is shown in figure 3. The top row shows seated clinic systolic and diastolic BP over time; the middle row the standing systolic BP and standing pulse rate, and the bottom row the orthostatic change in systolic BP and pulse rate. Change after chlorthalidone initiation was significant for seated and standing systolic $\mathrm{BP}$ only. At no time point was change statistically significant for diastolic BP, pulse rate, or orthostatic change in systolic BP or pulse rate. Orthostatic hypotension defined as systolic BP drop of $20 \mathrm{~mm} \mathrm{Hg}$ or more upon standing was seen in 5 subjects on 8 occasions. Five episodes occurred prior to starting chlorthalidone in 4 subjects. Among 3 subjects who each had one episode of orthostatic hypotension, 2 had orthostatic hypotension prior to starting chlorthalidone. In only 1 was orthostatic drop in $\mathrm{BP}$ accompanied by dizziness.

Changes in volume, target organs and laboratory tests are shown in figures 4 and 5 and table 1, respectively, and detailed in the online supplementary appendix.

\section{Adverse and Serious Adverse Events}

Adverse events were seen following administration of chlorthalidone in 7 subjects; 3 subjects had adverse events in the run-in period. After administration of chlorthalidone, 18 events were noted: hypokalemia $(\mathrm{n}=4)$, hyperuricemia (4), hyponatremia (3), transient elevations in serum creatinine (3), dizziness (2), hyperglycemia (1), and constipation (1). Before the administration of chlorthalidone, 3 events were noted: dizziness (1), urinary tract infection (1), and anemia requiring treatment with an erythropoietin-stimulating agent (1). In the case
Fig. 1. Individual and mean 24-hour ambulatory BP. Upper panel shows 24-hour, middle panel awake, and lower panel sleep systolic (left) and diastolic (right) BP. Each line represents an individual subject. Black triangles show the individual level of baseline $\mathrm{BP}$ in 2 subjects who did not have subsequent BP. Mean level of BP is shown by the circle, and whiskers represent one standard error of the mean. The table below the graph shows the number of participants at each time point (n), the duration (Durtn) of recording in minutes, the number of hours with at least one valid BP record- ing (Hrs), the change from baseline (CFB) in all 14 subjects (CFB14) or in the 12 completers (CFB12) and their $p$ values [CFB14 (p), CFB12 (p) derived from mixed models]. The \pm symbol reflects the standard error of the mean. The CFB in mean 24-hour SBP was significant [CFB14 $(\mathrm{p})=0.01$ ] at $10.5 \mathrm{~mm} \mathrm{Hg}$ using the mixed model in 14 subjects (CFB14); in 12 subjects who had paired measurements, the decline was also significant [CFB12 $(\mathrm{p})=0.05$ ] at $8.5 \mathrm{~mm} \mathrm{Hg}$ (CFB12). Declines were seen in awake and sleep systolic BP. Diastolic BP did not decline significantly.

(For fig. 1 see next page.) 

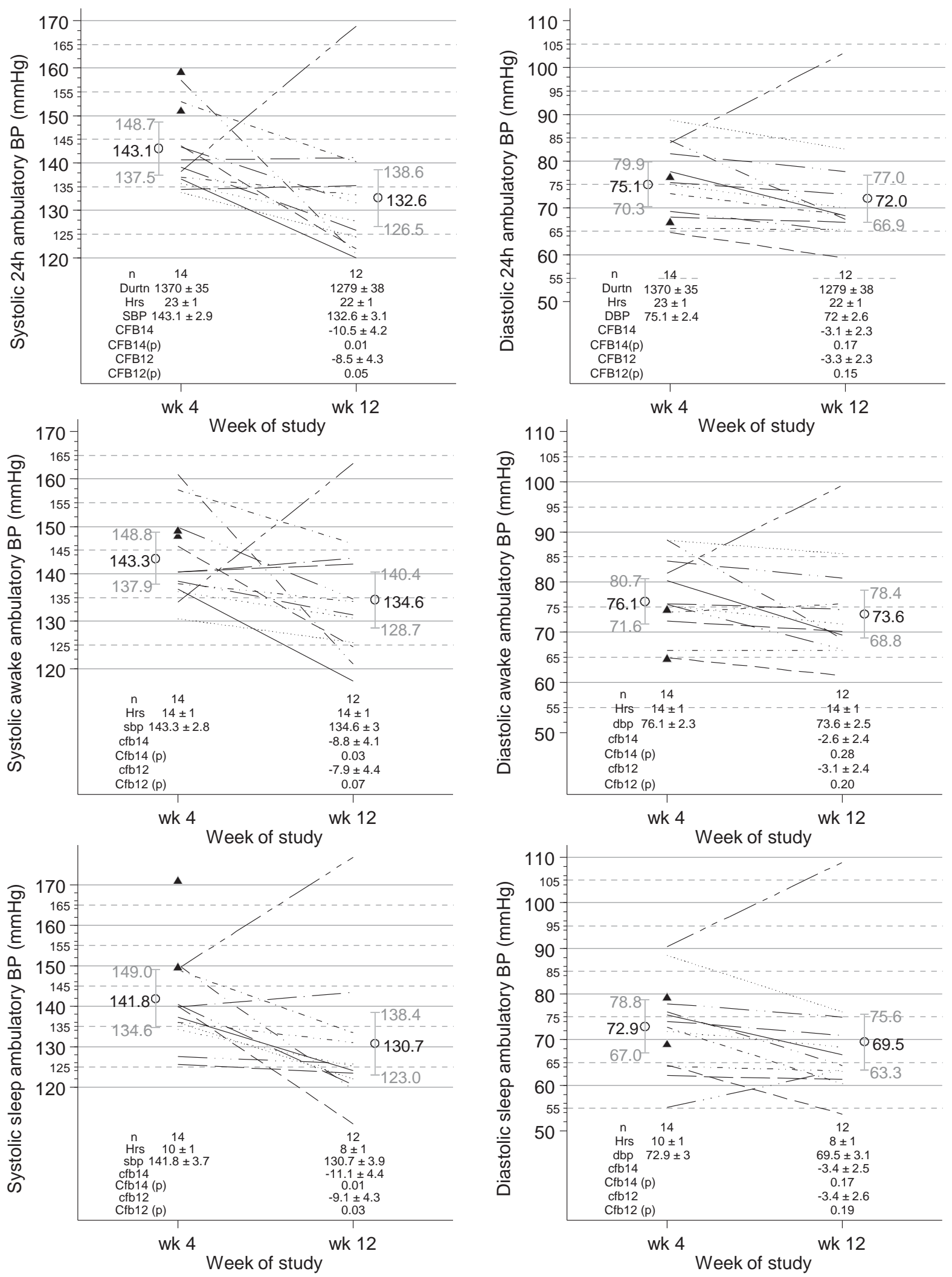
Fig. 2. Home BP at baseline and during the study. Upper graph shows the mean systolic BP and lower graph the mean diastolic BP over time. Circles show the mean and whiskers the standard error of the mean. Week -2 represents the baseline BP after which the usual medications were adjusted to a standard antihypertensive regimen. Two weeks after adjustment, at week 0 , chlorthalidone was initiated in a dose of $25 \mathrm{mg}$ once daily. Home BP was monitored at least every 4 weeks during the course of the study. The table below the graph shows the number of participants at each time point, the $\mathrm{CFB} \pm$ standard error $(\mathrm{SE})$ of the mean, the statistical significance of the change from baseline $[\mathrm{CFB}(\mathrm{p})]$, the change after drug initiation (CAD) and its statistical significance $[\mathrm{CAD}(\mathrm{p})]$. All changes measured either as change from baseline or change after drug initiation were statistically significant.

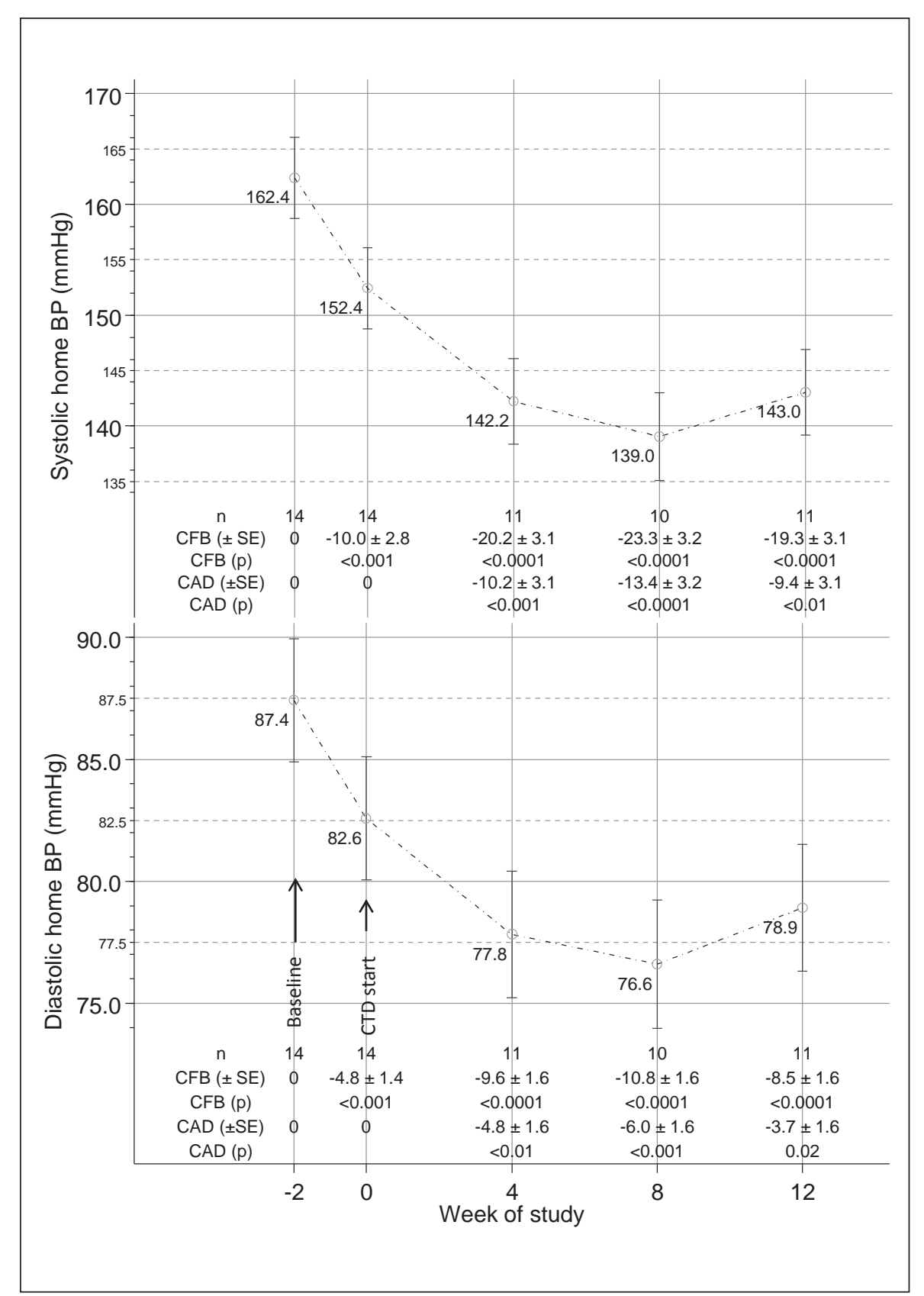

Fig. 3. Clinic oscillometric BP at baseline and during the study. Top row shows seated clinic systolic BP and diastolic BP over time, middle row the standing systolic BP and standing pulse rate, and the bottom row the orthostatic change in systolic BP and pulse rate. Circles show the mean and whiskers the standard error of the mean. Week -3 represents the first clinic visit at which home BP monitor was dispensed. Week -2 represents the baseline BP after which the usual medications were adjusted to a standard antihy- pertensive regimen. Two weeks after adjustment, at week 0 , chlorthalidone was initiated in a dose of $25 \mathrm{mg}$ once daily. Clinic BP both seated and upon standing for 1 min was monitored at baseline and at least every 4 weeks during the course of the study. The table below the graph shows the number of participants at each time point, the $\mathrm{CFB} \pm$ standard error (SE) of the mean, the CFB (p), the CAD and CAD (p).

(For fig. 3 see next page.) 

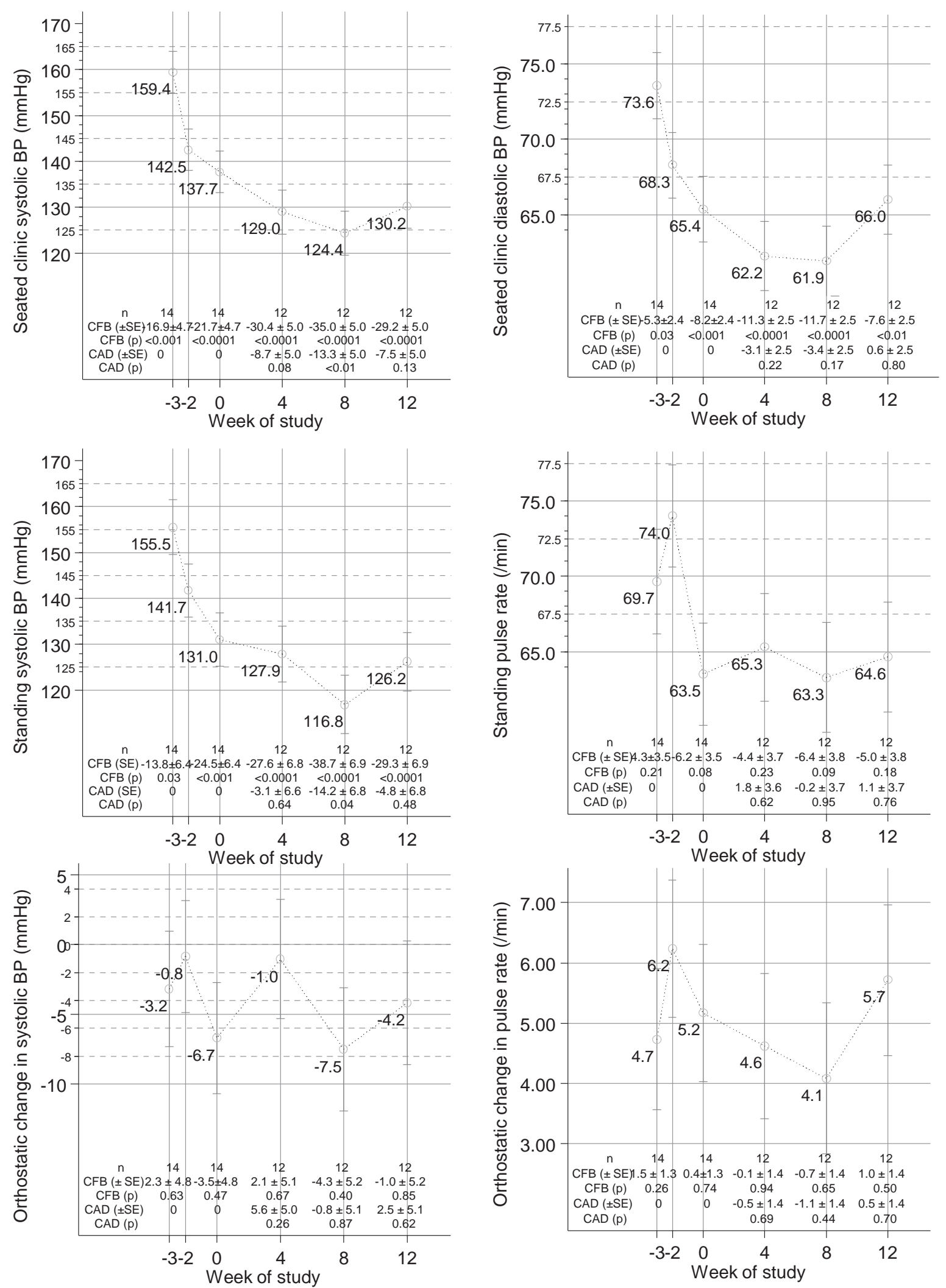

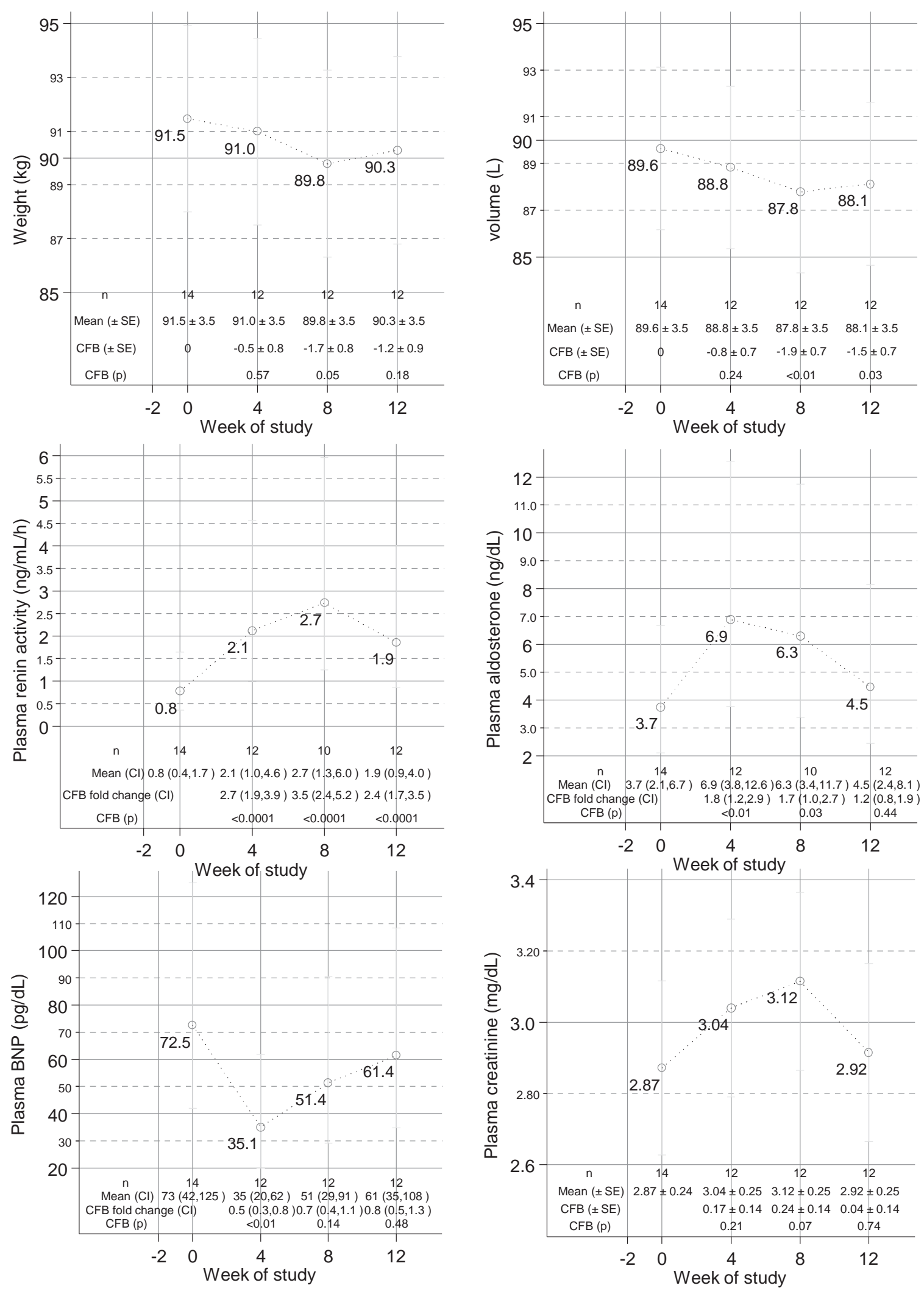

(For legend see next page.) 
of hypokalemia, onset was as early as 2 weeks and as late as 4 weeks. In 1 subject, hypokalemia persisted until week 8 . Similarly, hyperuricemia was seen by 6 weeks, and in 1 subject with a prior history of gout, it precipitated ankle pain thought to be gout. Hyperglycemia was seen within 2 weeks and transient elevations in serum creatinine between 2 and 6 weeks. Onset of dizziness in 2 subjects was at 2 and 4 weeks after chlorthalidone. Hyponatremia on the other hand occurred at 4-8 weeks. Counting all, 22 adverse events were recorded in 7 subjects after initiating chlorthalidone.

Two serious advents were noted. One subject following an elective kidney biopsy experienced a drop in hemoglobin from 12.1 to $10.3 \mathrm{~g} / \mathrm{dl}$ and was admitted for concern of internal bleeding. A computed tomographic scan showed no evidence for abdominal or renal bleed.

Table 1. Baseline and the time course of changes in blood chemistries

\begin{tabular}{|c|c|c|c|c|}
\hline Chemical component or $\mathrm{p}$ value & Baseline & Change at 4 weeks & Change at 8 weeks & Change at 12 weeks \\
\hline $\begin{array}{l}\text { Sodium, } \mathrm{mEq} / \mathrm{l} \\
\mathrm{p}\end{array}$ & $137.9(136.3,139.6)$ & $\begin{array}{l}-2.7(-4.4,-1.0) \\
<0.01\end{array}$ & $\begin{array}{l}-2.1(-3.8,-0.4) \\
0.01\end{array}$ & $\begin{array}{l}-1.4(-3.1,0.3) \\
0.11\end{array}$ \\
\hline $\begin{array}{l}\text { Potassium, mEq/l } \\
\mathrm{p}\end{array}$ & $4.31(4.04,4.57)$ & $\begin{array}{l}-0.26(-0.53,0.01) \\
0.06\end{array}$ & $\begin{array}{l}-0.05(-0.32,0.21) \\
0.69\end{array}$ & $\begin{array}{l}-0.00(-0.28,0.27) \\
0.98\end{array}$ \\
\hline $\begin{array}{l}\text { Bicarbonate, } \mathrm{mEq} / \mathrm{l} \\
\mathrm{p}\end{array}$ & $25.9(24.1,27.6)$ & $\begin{array}{l}0.38(-1.17,1.93) \\
0.63\end{array}$ & $\begin{array}{l}-0.12(-1.67,1.43) \\
0.88\end{array}$ & $\begin{array}{l}0.55(-1.00,2.10) \\
0.49\end{array}$ \\
\hline $\begin{array}{l}\text { Uric acid, mg/dl } \\
\mathrm{p}\end{array}$ & $7.27(6.07,8.47)$ & $\begin{array}{l}1.64(0.77,2.51) \\
<0.001\end{array}$ & $\begin{array}{l}1.64(0.77,2.51) \\
<0.001\end{array}$ & $\begin{array}{l}1.53(0.65,2.40) \\
<0.001\end{array}$ \\
\hline $\begin{array}{l}\text { Fasting glucose, } \mathrm{mg} / \mathrm{dl} \\
\mathrm{p}\end{array}$ & $146(97,196)$ & $\begin{array}{l}-3(-51,45) \\
0.91\end{array}$ & $\begin{array}{l}30(-18,77) \\
0.22\end{array}$ & $\begin{array}{l}10(-37,58) \\
0.67\end{array}$ \\
\hline $\begin{array}{l}\text { Calcium, mg/dl } \\
\mathrm{p}\end{array}$ & $8.89(8.60,9.17)$ & $\begin{array}{l}-0.10(-0.34,0.13) \\
0.39\end{array}$ & $\begin{array}{l}0.13(-0.11,0.37) \\
0.29\end{array}$ & $\begin{array}{l}0.04(-0.20,0.27) \\
0.76\end{array}$ \\
\hline $\begin{array}{l}\text { Phosphorus, mg/dl } \\
\mathrm{p}\end{array}$ & $3.99(3.65,4.32)$ & $\begin{array}{l}0.23(-0.18,0.63) \\
0.27\end{array}$ & $\begin{array}{l}-0.11(-0.51,0.29) \\
0.6\end{array}$ & $\begin{array}{l}0.03(-0.38,0.44) \\
0.89\end{array}$ \\
\hline $\begin{array}{l}\text { Magnesium, mg/dl } \\
\mathrm{p}\end{array}$ & $2.00(1.78,2.22)$ & $\begin{array}{l}0.30(0.06,0.55) \\
0.02\end{array}$ & $\begin{array}{l}0.18(-0.06,0.43) \\
0.14\end{array}$ & $\begin{array}{l}0.12(-0.13,0.36) \\
0.34\end{array}$ \\
\hline $\begin{array}{l}\text { Parathryoid hormone-intact, } \mathrm{pg} / \mathrm{ml} \\
\mathrm{p}\end{array}$ & $114.2(71.9,181.4)$ & & & $\begin{array}{l}-0.10(-0.32,0.13) \\
0.4\end{array}$ \\
\hline $\begin{array}{l}\text { Cholesterol, mg/dl } \\
\mathrm{p}\end{array}$ & $151.7(130.4,173.1)$ & & & $\begin{array}{l}-3.4(-32.6,25.8) \\
0.82\end{array}$ \\
\hline $\begin{array}{l}\text { Hemoglobin A1C, \% } \\
\text { p }\end{array}$ & $7.56(6.47,8.66)$ & & & $\begin{array}{l}0.41(-0.45,1.27) \\
0.35\end{array}$ \\
\hline
\end{tabular}

$\mathrm{p}$ values reflect the change from baseline. Values in parentheses represent minimum and maximum.

Fig. 4. Markers of volume at baseline and during the study. Top panel shows measurements by BodPod of weight and total body volume. Maximal changes in both of these measures were seen at 8 weeks. Middle panel shows seated plasma renin activity and plasma aldosterone. Renin was 2.7 -fold higher within 4 weeks of starting chlorthalidone and was maximally stimulated at 8 weeks. Aldosterone on the other hand was maximally stimulated at 4 weeks and remained stimulated at 8 weeks. However, at 12 weeks, aldosterone was not different compared to baseline. Bottom panel:
BNP was half that of the baseline at 4 weeks, but gradually returned to baseline. Plasma creatinine was changed maximally by $0.24 \mathrm{mg} /$ $\mathrm{dl}$ at 8 weeks but also returned toward baseline at 12 weeks. The table below the graph shows the number of participants at each time point, the $\mathrm{CFB} \pm$ standard error (SE) of the mean or the $95 \%$ confidence interval of the fold change in case of renin, aldosterone, and BNP. The statistical significance of the change from baseline is in row labeled CFB (p). 


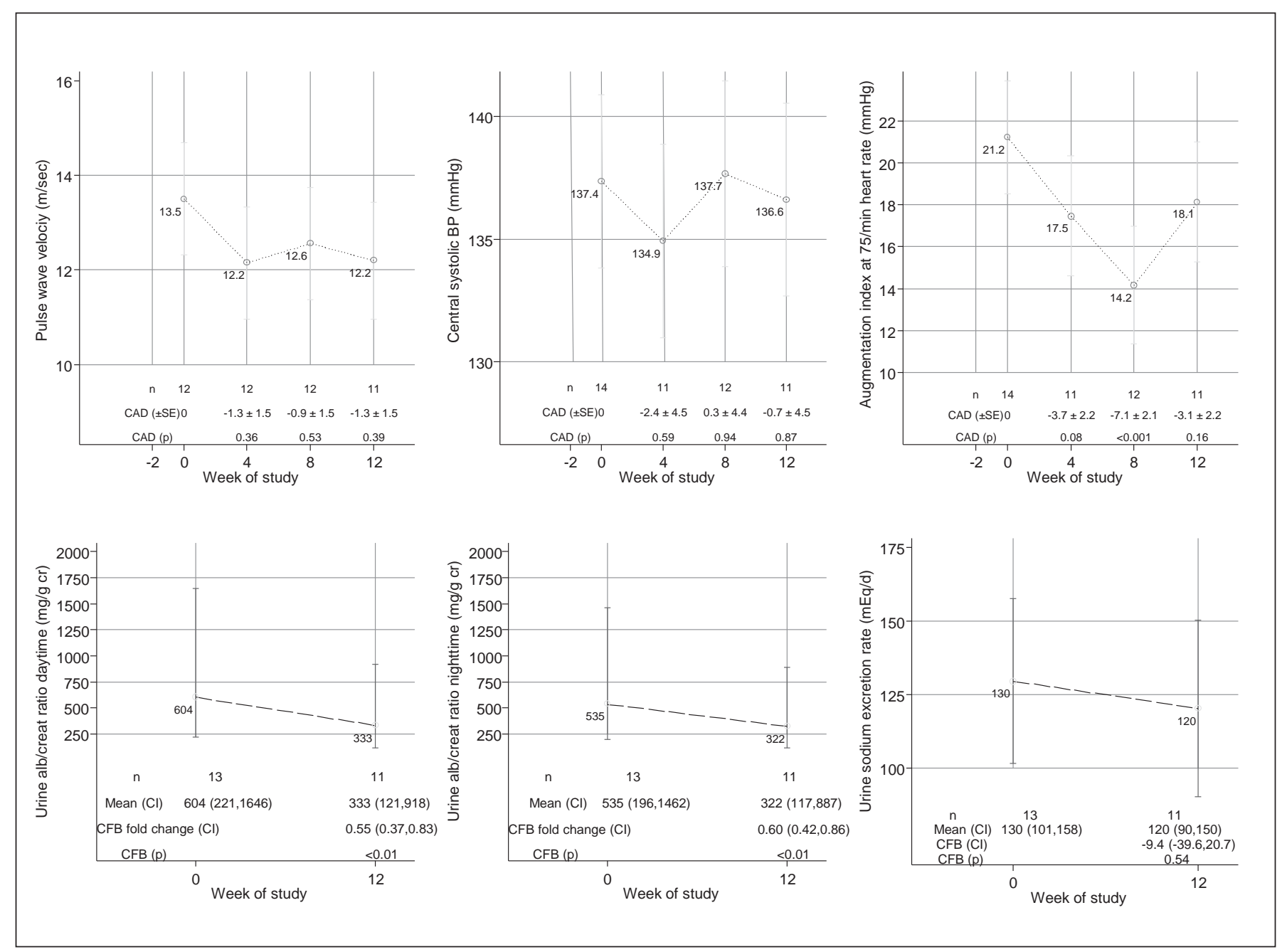

Fig. 5. Markers of target organ damage at baseline and during the study. The top panel shows carotid-to-femoral pulse wave velocity, central systolic BP, and augmentation index corrected to heart rate at 75 beats $/ \mathrm{min}$. The bottom panel shows the results of urinary excretion of albumin during the day and night as well as 24-hour urinary sodium excretion rate at baseline and at 12 weeks of study. creat and $\mathrm{cr}=$ Creatinine.

The other subject, a 59-year-old black man with multiple strokes in the past, diabetes mellitus, and history of seizures, had a new-onset ischemic stroke. He at a later date contributed to 4 more serious adverse events: hypotension on 2 occasions and syncope on another 2 occasions. This subject volitionally stopped all antihypertensive medications, did not measure $\mathrm{BP}$ at home beyond 4 weeks and was the only one to experience a rise from baseline in systolic and diastolic ambulatory BP at 12 weeks.

Given the pilot nature of the study and the high likelihood of false discovery, subgroup analyses were not performed.

\section{Discussion}

Among subjects with moderate to advanced CKD and uncontrolled treated hypertension, the major findings of the study are as follows. (1) Chlorthalidone therapy may lower BP predominantly systolic over a few weeks. (2) In contrast to clinic BP measurements, the BP-lowering effects of therapy were better detected by out-of-office BP monitoring; home BP monitoring showed significant improvement in both systolic and diastolic BP, whereas clinic BP monitoring could not detect improvement in diastolic BP. (3) Lowering of BP is likely evoked by lowering of extracellular fluid volume (as shown by lowering of 
total body volume, lowering of B-type natriuretic peptide (BNP), and an increase in renin and aldosterone concentration); mitigation of these effects over time suggests nonvolume mechanisms such as lowering of vascular resistance maintaining the BP-lowering effect. (4) Side effects such as hypokalemia, hyponatremia, hyperuricemia, transient expected changes in serum creatinine concentration, and orthostatic hypotension are common; such adverse effects are likely to be seen within a few weeks of starting drug therapy and therefore close monitoring is essential. The occurrence of these side effects further confirms the biological activity of chlorthalidone in advanced CKD. Our data support evidence from earlier studies, albeit of small size and short durations, that thiazide and thiazide-like diuretics can indeed be effective in people with moderate to advanced CKD (see online suppl. appendix for details) [10-14].

Our study adds to the existing knowledge of the use of thiazides in advanced CKD in several ways. First, although the duration of intervention was 12 weeks, the study was the longest reported. Second, it is the only study to our knowledge that evaluated the effect of thiazides on $\mathrm{BP}$ with the use of the reference standard of 24-hour ambulatory BP monitoring. Third, the study evaluated the mechanisms of BP reduction. To our knowledge, this is the first time that the measurement of total body volume has been evaluated in a clinical trial with diuretics. It offers an objective way of evaluating total body volume, and we demonstrated highly significant reduction in total body volume within weeks of starting chlorthalidone. Coupled with reduction in BNP early and renin/aldosterone a few weeks later, it provides a strong argument that these drugs probably act by reducing plasma volume. Fourth, the reduction in albumin excretion rate during awake and asleep hours suggests that this drug has the potential to abrogate target organ damage and potentially protect the kidney and the cardiovascular system in the long term [15]. Fifth, the intense monitoring of subjects every 4 weeks revealed that the drug is not without adverse effects. It is clear that using this drug will require close monitoring of $\mathrm{BP}$, renal function, electrolytes, glycemia, and gout.

There are several limitations to this study. First, it was limited to a small number of subjects and there was no placebo group. This design was deliberate given that the intent of the study was to generate preliminary data to design a future randomized trial. Second, the study included 4 subjects with stage 3B CKD. In such subjects, thiazides or thiazide-like diuretics are generally recommended. However, 8 of the 12 subjects completing the study had estimated GFR $<30 \mathrm{ml} / \mathrm{min} / 1.73 \mathrm{~m}^{2}$, a stage of kidney disease where thiazide diuretics are generally considered futile.

In summary, this pilot study addresses an important question of how to treat poorly controlled hypertension in CKD with an established but a poorly utilized drug better. At present, guidelines do not recommend the use of thiazides in patients with advanced CKD. This pilot study suggests that chlorthalidone may improve BP control in patients with moderately advanced CKD, but it might also cause adverse effects. These data may help design a double-blind, placebo-controlled, randomized trial in patients with CKD and poorly controlled hypertension. Only then can we evaluate the true risks and benefits of chlorthalidone such that its place in the management of hypertension in subjects with CKD can be established.

\section{Acknowledgments}

We thank Megan Roadley, Opeyemi Olorungbounmi and America Newnum for technical assistance in the study.

This work was supported by the Indiana Institute for Medical Research.

\section{Disclosure Statement}

None.

\section{References}

Coresh J, Wei GL, McQuillan G, Brancati FL, Levey AS, Jones C, Klag MJ: Prevalence of high blood pressure and elevated serum creatinine level in the United States: findings from the third National Health and Nutrition Examination Survey (1988-1994). Arch Intern Med 2001;161:1207-1216.
Vasavada N, Agarwal R: Role of excess volume in the pathophysiology of hypertension in chronic kidney disease. Kidney Int 2003;64: 1772-1779.

3 http://www.kidney.org/professionals/kdoqi/ guidelines_bp/guide_12.htm.
Chobanian AV, Bakris GL, Black HR, Cushman WC, Green LA, Izzo JL Jr, Jones DW, Materson BJ, Oparil S, Wright JT Jr, Roccella EJ: Seventh report of the Joint National Committee on Prevention, Detection, Evaluation, and Treatment of High Blood Pressure. Hypertension 2003;42:1206-1252. 
5 Agarwal R, Sinha AD: Thiazide diuretics in advanced chronic kidney disease. J Am Soc Hypertens 2012;6:299-308.

6 6 Levey AS, Stevens LA, Schmid CH, Zhang YL, Castro AF III, Feldman HI, Kusek JW, Eggers P, Van Lente F, Greene T, Coresh J: A new equation to estimate glomerular filtration rate. Ann Intern Med 2009;150:604-612.

7 Agarwal R, Lewis RR, Davis JL, Becker B: Lisinopril therapy for hemodialysis hypertension - hemodynamic and endocrine responses. Am J Kidney Dis 2001;38:1245-1250.

-8 O'Brien E, Asmar R, Beilin L, Imai Y, Mallion JM, Mancia G, Mengden T, Myers M, Padfield P, Palatini P, Parati G, Pickering T, Redon J, Staessen J, Stergiou G, Verdecchia P: European Society of Hypertension recommendations for conventional, ambulatory and home blood pressure measurement. J Hypertens 2003;21:821-848.
9 Holden JE, Kelley K, Agarwal R: Analyzing change: a primer on multilevel models with applications to nephrology. Am J Nephrol 2008;28:792-801.

10 Fliser D, Schroter M, Nerubeck M, Ritz E: Coadministration of thiazides increases the efficacy of loop diuretics even in patients with advanced renal failure. Kidney Int 1994;46: 482-488.

11 Wollam GL, Tarazi RC, Bravo EL, Dustan HP: Diuretic potency of combined hydrochlorothiazide and furosemide therapy in patients with azotemia. Am J Med 1982;72:929-938.
12 Knauf H, Mutschler E: Diuretic effectiveness of hydrochlorothiazide and furosemide alone and in combination in chronic renal failure. J Cardiovasc Pharmacol 1995;26:394-400.

13 Dussol B, Moussi-Frances J, Morange S, Somma-Delpero C, Mundler O, Berland Y: A randomized trial of furosemide vs hydrochlorothiazide in patients with chronic renal failure and hypertension. Nephrol Dial Transplant 2005;20:349-353.

14 Dussol B, Moussi-Frances J, Morange S, Somma-Delpero C, Mundler O, Berland Y: A pilot study comparing furosemide and hydrochlorothiazide in patients with hypertension and stage 4 or 5 chronic kidney disease. J Clin Hypertens (Greenwich) 2012;14:32-37.

15 Vogt L, Waanders F, Boomsma F, de Zeeuw D, Navis G: Effects of dietary sodium and hydrochlorothiazide on the antiproteinuric efficacy of losartan. J Am Soc Nephrol 2008; 19: 999-1007. 